\title{
Heart Rate Turbulence and Clinical Prognosis in Hypertrophic Cardiomyopathy and Myocardial Infarction
}

\author{
Tatsuya Kawasaki, MD; Akihiro Azuma, MD*; Satoshi Asada, MD; \\ Mitsuyoshi Hadase, MD; Tadaaki Kamitani, MD; Shingo Kawasaki, MD; \\ Toshiro Kuribayashi, MD**; Hiroki Sugihara, MD
}

\begin{abstract}
Short-term fluctuations in sinus cycle length after a single ventricular premature complex (VPC) have attracted considerable interest and has been termed heart rate turbulence (HRT). The onset and slope of HRT have each been reported to be independent and powerful predictors of clinical prognosis in patients with myocardial infarction (MI), but there are no data available for patients with hypertrophic cardiomyopathy (HCM). Thus the present study analyzed the 2 HRT variables to determine their prognostic value in HCM patients. Holter monitoring data were obtained from $104 \mathrm{HCM}$ patients, $44 \mathrm{MI}$ patients and 56 normal controls, from which singular VPCs followed by $\geq 20$ normal sinus beats were isolated and the HRT onset and slope were automatically calculated. HRT onset and slope were abnormal in MI patients, but not in HCM patients, as compared with normal control subjects (onset $-1.1 \pm 2.9,-2.1 \pm 3.4,-1.4 \pm 5.1 \%$; slope $10.6 \pm 8.6,18.0 \pm 13.9,16.6 \pm 9.7 \mathrm{~ms} /$ beat, respectively). During the follow-up period of $27 \pm 10$ months, $7 \mathrm{HCM}$ patients and $10 \mathrm{MI}$ patients either died from cardiac death or were hospitalized for congestive heart failure. In MI patients, HRT onset was higher and the HRT slope was lower in patients with cardiac events than in patients without (onset $1.1 \pm 2.7$ vs $-1.7 \pm 2.7 \%, \mathrm{p}=0.011$; slope $5.7 \pm 4.3$ vs $12.0 \pm 9.0 \mathrm{~ms} /$ beat, $\mathrm{p}=0.028$ ). In HCM patients, however, the HRT onset and slope were similar between patients with and without cardiac events (onset $-2.0 \pm 2.0$ vs $-2.1 \pm 3.5 \%, p=0.98$; slope $18.1 \pm 10.9$ vs $18.0 \pm$ $14.0 \mathrm{~ms} /$ beat, $\mathrm{p}=0.68$ ). In conclusion, unlike MI patients, the HRT variables in selected HCM patients were not abnormal and failed to predict the clinical prognosis. (Circ J 2003; 67: 601-604)
\end{abstract}

Key Words: Heart rate turbulence; Hypertrophic cardiomyopathy; Prognosis; Ventricular premature complex

$\mathbf{H}$ eart rate turbulence (HRT) represents short-term fluctuations in sinus cycle length after a single ventricular premature complex (VPC). Normally, HRT comprises sinus rate acceleration during several beats immediately following a VPC and the subsequent deceleration back to the baseline before the 20th beat ${ }^{1,2}$ It is now believed that HRT is principally triggered by underlying physiological alterations of cardiac autonomic regulation; a VPC preceded by a short coupling interval results in a sudden drop in blood pressure because of insufficient ventricular filling, with subsequent transient loss of vagal efferent activity leading to sinus rate acceleration?,4

The early acceleration and late deceleration in sinus cycle length following a VPC are termed the HRT onset and HRT slope, respectively, and are each currently being used in the assessment of HRT. Recently, these variables were reported to be powerful independent predictors of the clinical prognosis in patients with myocardial infarction (MI) 5-7 $^{-7}$ However, data for patients with hypertrophic cardiomyopathy (HCM) are not available, so the purpose of the present

(Received February 18, 2003; revised manuscript received April 8, 2003; accepted April 25, 2003)

Department of Cardiology, Matsushita Memorial Hospital, Osaka, *Second Department of Medicine, Kyoto Prefectural University of Medicine, Kyoto and **Kuribayashi Clinic of Cardiology, Fukuoka, Japan

Mailing address: Tatsuya Kawasaki, MD, Department of Cardiology, Matsushita Memorial Hospital, 5-55 Sotojima, Moriguchi, Osaka 570-

8540, Japan. E-mail: js-k@wf6.so-net.ne.jp study was to determine these variables in HCM patients and correlate them with the clinical prognosis.

\section{Methods}

Study Population

We studied 104 consecutive ambulatory HCM patients, 44 MI patients and 56 normal controls who were treated at or referred to Matsushita Memorial Hospital during the period of April 2000 to March 2002. All subjects had regular sinus rhythm and were clinically stable. The MI patients and controls were matched to the HCM patients with regard to age and gender distribution. The diagnosis of HCM was based on echocardiographic demonstration of left ventricular (LV) hypertrophy with end-diastolic wall thickness $\geq 15 \mathrm{~mm}$ and LV end-diastolic dimensions $\leq 55 \mathrm{~mm}$, in the absence of any cardiac or systemic disorder that causes LV hypertrophy, 89 The HCM patient group consisted of 83 men and 21 women with a mean age of $56 \pm 13$ years; of them 8 had apical hypertrophy, 12 had a family history of sudden death, 17 had episodes of syncope, 34 had ventricular tachycardia defined as 3 or more consecutive VPCs with a frequency $>120$ beats $/$ min. The maximum LV wall thickness was $22 \pm 3 \mathrm{~mm}$ and LV fractional shortening was $41 \pm 8 \%$ on echocardiogram. Eight patients had a Dopplercalculated resting left ventricular outflow tract gradient of at least $30 \mathrm{mmHg}$. The class of heart failure was New York Heart Association (NYHA) I in 65 patients, II in 38 patients and III in 1 patient. 
Table 1 Baseline Characteristics and Heart Rate Turbulence (HRT) Variables in the Study Subjects

\begin{tabular}{|c|c|c|c|c|}
\hline & Hypertrophic cardiomyopathy & Myocardial infarction & Control & $p$ value \\
\hline No. of subjects & 102 & 43 & 53 & \\
\hline Age (years) & $56 \pm 13$ & $58 \pm 10$ & $55 \pm 15$ & 0.21 \\
\hline Male $(\%)$ & $82(80)$ & $34(79)$ & $42(79)$ & 0.98 \\
\hline Left ventricular fractional shortening (\%) & $41 \pm 8$ & $22 \pm 10 *$ & $38 \pm 6$ & $<0.0001$ \\
\hline HRT onset $(\%)$ & $-2.1 \pm 3.4$ & $-1.1 \pm 2.9$ & $-1.4 \pm 5.1$ & 0.22 \\
\hline HRT slope (ms/beat) & $18.0 \pm 13.9$ & $10.6 \pm 8.6^{*}$ & $16.6 \pm 9.7$ & 0.00084 \\
\hline Abnormal HRT onset, $\geq 0 \%(\%)$ & $20(20)$ & $15(35)$ & $10(19)$ & 0.099 \\
\hline Abnormal HRT slope, $\leq 2.5 \mathrm{~ms} /$ beat $(\%)$ & $3(3)$ & $6(14) *$ & $0(0)$ & 0.0026 \\
\hline
\end{tabular}

$* p<0.01$ vs hypertrophic cardiomyopthy or control

All MI patients had a documented history of acute MI or electrocardiographic (ECG) evidence of MI (ie, deep Qwave). The MI patient group consisted of 34 men and 10 women with a mean age of $58 \pm 10$ years; ventricular tachycardia was observed in 11 patients; the LV ejection fraction (LVEF) by venticulogram was $32 \pm 13 \%$; and LV fractional shortening was $22 \pm 10 \%$ on echocardiogram; NYHA class was I in 10, II in 29 and III in 5 patients.

Fifty-six healthy subjects served as normal controls;

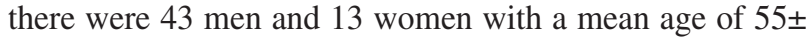
15 years; none had any cardiovascular symptoms or history of cardiovascular disease; all exhibited normal 12-lead ECG and echocardiographic findings; LV fractional shortening was $38 \pm 6 \%$ on echocardiogram.

\section{HRT Analysis}

All study subjects underwent 24-h Holter monitoring. After a manual review of the recorded data, we selected singular VPCs preceded and followed by $\geq 20$ normal sinus beats! 10 The 20 subsequent R-R intervals in the beats following a VPC were measured automatically and the HRT onset and slope were calculated according to the original method reported by Schmidt et al5 Briefly, HRT onset was defined as the difference between the mean of the first 2 sinus-rhythm R-R intervals following the compensatory pause after a VPC and the mean of the last 2 sinus-rhythm $\mathrm{R}-\mathrm{R}$ intervals preceding the VPC, expressed as a percentage of the former. The HRT slope was defined as the maximum positive slope of a regression line assessed over any sequence of 5 subsequent sinus-rhythm R-R intervals within the first 20 sinus-rhythm intervals after a VPC, expressed as $\mathrm{ms} /$ beat. The HRT onset or slope was defined as abnormal if the onset was $\geq 0 \%$ or the slope was $\leq 2.5 \mathrm{~ms} /$ beat 5 Of all the subjects in this study, $2 \mathrm{HCM}$ patients, $1 \mathrm{MI}$ patient and 3 controls were excluded from the analysis because a VPC could not be found on their Holter recordings.

\section{Follow-up and End-point}

All subjects were followed up, beginning with 24-h Holter ambulatory ECG monitoring. Patient information was obtained from available medical records and interviews with the patients and/or their physicians in charge. Followup data were available for all patients. The efficacy endpoint was the incidence of cardiac events: cardiac death or heart failure. Cardiac death was defined as death from myocardial infarction, heart failure or arrhythmia, or sudden death. Sudden death was defined as instantaneous and unexpected death within $1 \mathrm{~h}$ of the onset of symptoms or an unwitnessed death in a subject previously in a stable condition. Episodes of successfully resuscitated life-threatening arrhythmia were classified as cardiac death. Heart failure was defined as requiring hospitalization for initiation of intravenous treatment with inotropic, vasodilator, and/or diuretics; mechanical ventilation or circulatory support.

\section{Statistical Analysis}

Categorical variables were reported as absolute (percentages), and compared by chi-square test or Fisher's exact test. Continuous variables were expressed as the mean \pm standard deviation. Age was compared between the 3 groups by one-way analysis of variance followed by Scheffe's multiple comparison test. Because the distributions of the HRT slope and onset were extremely skewed, these variables between 2 groups or 3 groups were assessed using the Mann-Whitney U test or Kruskal-Wallis test, respectively. The reproducibility of HRT onset or HRT slope was tested on 2 random VPCs in 50 HCM patients. The Spearman rank correlation coefficient was used to compare the trend analysis. Identification of predictors of cardiac events was based on Cox proportional hazards analysis as risk ratio and $95 \%$ confidence interval. A $\mathrm{p}<0.05$ was considered statistically significant.

\section{Results}

Age and gender distribution were similar for the 3 groups (Table 1). The LV fractional shortening was lower in MI patients than in HCM patients or controls. HRT onset did not differ among the 3 groups, but the incidence of abnormal onset tended to be higher in MI patients than in HCM patients or controls $(35 \%, 20 \%$, and $19 \%$, respectively; $\mathrm{p}=0.099$ ). The HRT slope was lower in MI patients than in HCM patients or controls $(10.6 \pm 8.6,18.0 \pm 13.9$, and $16.6 \pm 9.7 \mathrm{~ms} / \mathrm{beat}$, respectively; $\mathrm{p}=0.00084)$. Similarly, the incidence of abnormal HRT slope was more frequent in MI patients than in HCM patients or controls $(14 \%, 3 \%, 0 \%$, respectively; $\mathrm{p}=0.0026)$. There were no significant differences in any of the 4 HRT variables between HCM patients and controls.

There was a significant correlation in the HRT onset $(\mathrm{r}=0.63, \mathrm{p}=0.0006$, mean differences $0.1 \pm 4.3)$ and HRT slope $(r=0.68, p<0.0001$, mean differences $2.6 \pm 9.8)$ between measurements made for different VPCs. The concordance rate of abnormal HRT onset or HRT slope was $96 \%$ or $88 \%$, respectively.

During the follow-up period of $27 \pm 10$ months, cardiac events occurred in $7 \mathrm{HCM}$ patients and $10 \mathrm{MI}$ patients, but not in any of the controls. The follow-up period in each group was similar $(27 \pm 9,28 \pm 11,26 \pm 10$ months, respectively). In the HCM group, 1 patient died of progressive heart failure and 6 were hospitalized for congestive heart failure. In the MI group, 1 patient died suddenly from ventricular fibrillation, 5 died of progressive heart failure and 4 were hospitalized for congestive heart failure. 
Table 2 Heart Rate Turbulence (HRT) and Outcome in Patients With Hypertrophic Cardiomyopathy or Myocardial Infarction

\begin{tabular}{|c|c|c|c|c|c|c|}
\hline & \multicolumn{3}{|c|}{ HRT variables according to cardiac events } & \multicolumn{3}{|c|}{ Association with time to cardiac events } \\
\hline & Events (+) & Events (-) & $p^{*}$ value & Risk ratio & $95 \% C I$ & $p^{\dagger}$ value \\
\hline \multicolumn{7}{|l|}{ Myocardial infarction } \\
\hline HRT onset (\%) & $1.1 \pm 2.7$ & $-1.7 \pm 2.7$ & 0.011 & 1.16 & $0.98-1.37$ & 0.086 \\
\hline HRT slope (ms/beat) & $5.7 \pm 4.3$ & $12.0 \pm 9.0$ & 0.028 & 0.83 & $0.71-0.98$ & 0.024 \\
\hline Abnormal HRT onset, $\geq 0 \%$ (\%) & $6(67)$ & $9(26)$ & 0.025 & 3.76 & $0.94-15.15$ & 0.061 \\
\hline Abnormal HRT slope, $\leq 2.5$ ms/beat (\%) & $4(44)$ & $2(6)$ & 0.012 & 5.65 & $1.37-23.25$ & 0.016 \\
\hline \multicolumn{7}{|l|}{ Hypertrophic cardiomyopathy } \\
\hline HRT onset (\%) & $-2.0 \pm 2.0$ & $-2.1 \pm 3.5$ & 0.98 & 1.03 & $0.83-1.27$ & 0.79 \\
\hline HRT slope (ms/beat) & $18.1 \pm 10.9$ & $18.0 \pm 14.0$ & 0.68 & 1.00 & $0.95-1.06$ & 0.99 \\
\hline Abnormal HRT onset, $>0 \%$ (\%) & $1(13)$ & $19(20)$ & $>0.99$ & 0.46 & $0.055-3.86$ & 0.48 \\
\hline Abnormal HRT slope, $\leq 2.5 \mathrm{~ms} /$ beat (\%) & $0(0)$ & $3(3)$ & $>0.99$ & - & - & - \\
\hline
\end{tabular}

CI, confidence interval. ${ }^{\star}$ Mann-Whitney U test or chi-square test; ${ }^{\dagger}$ univariate Cox proportional hazards analysis.

Table 2 lists the incidence of patients with abnormal values of HRT onset and HRT slope and their mean values in relation to cardiac events in patients with HCM or MI. In MI patients, the HRT onset was higher and the slope was lower in patients with cardiac events than in those without (onset $1.1 \pm 2.7$ vs $-1.7 \pm 2.7 \%, \mathrm{p}=0.011$; slope $5.7 \pm 4.3$ vs $12.0 \pm 9.0 \mathrm{~ms} / \mathrm{beat}, \mathrm{p}=0.028)$. Furthermore, the incidence of abnormal HRT onset and slope were more frequent in patients with events (onset $67 \%$ vs $26 \%, \mathrm{p}=0.025$; slope $44 \%$ vs $6 \%, \mathrm{p}=0.012$ ). On univariate Cox proportional hazards analysis, HRT slope and abnormal HRT slope were each predictive of cardiac events in MI patients. In the HCM patients, however, HRT onset and slope were similar between patients with and without cardiac events (onset $-2.0 \pm 2.0$ vs $-2.1 \pm 3.5 \%, p=0.98$; slope $18.1 \pm 10.9$ vs $18.0 \pm$ $14.0 \mathrm{~ms} /$ beat, $\mathrm{p}=0.68)$. Furthermore, the incidence of abnormal HRT onset and HRT slope were similar between patients with or without cardiac events (onset $13 \%$ vs $20 \%$, p $>0.99$; slope $0 \%$ vs $3 \%, p>0.99$ ), and were not predictive of cardiac events on Cox proportional hazards analysis.

\section{Discussion}

The major finding of this study was that HRT variables remain normal and are not predictive of clinical prognosis in HCM patients. In MI patients, on the other hand, the HRT variables were abnormal and of prognostic value, as reported previously5,6 We may safely consider that the present results are valid to some extent despite the following limitations. First, the subject number was relatively small and the follow-up period was not long, with a few patients dying during this period. A larger number of patients and longer study duration are needed to resolve these problems. Second, selection bias may be present; that is, the exclusion of patients with no VPC at all in their Holter records for whom HRT could not be determined. However, such patients are generally at low risk and no cardiac events occurred in the patients that did not have a VPC on their Holter monitoring. Third, cardioactive drugs, especially $\beta$-blockers and/or antiarrhythmic drugs, may have modified the results, although only a few of the HCM patients were taking $\beta$-blockers $(8 \%)$ or antiarrhythmic drugs (class IA $12 \%$; class IB 10\%; class IC $1 \%$ ), and their exclusion from the analysis did not alter the results.

The change to HRT is thought to depend, in part, on abnormal autonomic nerve activity. Electrophysiologic studies have demonstrated that the change in HRT onset and slope is provoked by vagal efferent blockade with atropine, but not by sympathetic blockade with esmolol2,11 Furthermore, Lin et al, ${ }^{11}$ by systematically inducing VPC in patients without structural heart disease, demonstrated that HRT onset correlated negatively, and HRT slope correlated positively, with baroreflex sensitivity. A recent study of 45 patients with congestive heart failure mainly caused by coronary artery disease or idiopathic dilated cardiomyopathy has shown that the change to HRT correlated well with attenuated baroreflex sensitivity! 10 Therefore, attenuated baroreflex sensitivity may evoke the change to HRT via the vagal efferent limb of the baroreflex arch.

In the present HCM patients, however, the HRT variables were similar to those of the controls and hence baroreflex sensitivity may not have been impaired although we did not measure this parameter. Thomson et al ${ }^{12}$ investigated baroreflex function in $29 \mathrm{HCM}$ patients and 32 normal controls, and reported that integrated baroreceptor sensitivity and carotid artery baroreceptor sensitivity were similar between the 2 groups, which supports our results. However, they also studied cardiopulmonary baroreceptor sensitivity, assessed by measuring forearm vascular resistance during lower body negative pressure, and reported that cardiopulmonary baroreceptor sensitivity was reduced in patients with HCM. The discrepancy between these 2 studies of HCM patients may in part be explained by the difference in method of baroreflex function assessment or by differences in the clinical features of patients. Integrated baroreceptor sensitivity and carotid artery baroreceptor sensitivity are variables based on the change in RR intervals, whereas cardiopulmonary baroreceptor sensitivity does not. In the other reports ${ }^{10,11}$ that linked baroreflex function to HRT, baroreceptor sensitivity was assessed by the method based on the R-R intervals. Furthermore, there was a history of syncope or presyncope in $79 \%$ of patients in the study by Thomson et al, whereas the incidence was $16 \%$ of the present patients. Thomson et al reported that cardiopulmonary baroreceptor sensitivity was more impaired in patients with syncope than in patients without syncope. In that regard, a head-up tilt test of $36 \mathrm{HCM}$ patients by Gilligan et $\mathrm{al}^{13}$ showed that hypotension caused by an abnormal baroreflex occurred frequently in patients with a history of syncope, but rarely in patients with no syncopal symptoms.

In conclusion, although HRT variables are abnormal and of prognostic value in MI patients, these variables remained normal and failed to predict clinical prognosis in selected HCM patients. These findings need to be confirmed in a larger prospective study. 


\section{Acknowledgments}

The authors gratefully acknowledge the expert technical assistance of T Takahasi, E Hiramatsu, J Murase and K Higashiyama.

\section{References}

1. Watanabe MA, Marine JE, Sheldon R, Josephson ME. Effects of ventricular premature stimulus coupling interval on blood pressure and heart rate turbulence. Circulation 2002; 106: 325-330.

2. Marine JE, Watanabe MA, Smith TW, Monahan KM. Effect of atropine on heart rate turbulence. Am J Cardiol 2002; 89: 767-769.

3. Wichterle D, Melenovsky V, Malik M. Mechanisms involved in heart rate turbulence. Card Electrophysiol Rev 2002; 6: 262-266.

4. Mrowka R, Persson PB, Theres H, Patzak A. Blunted arterial baroreflex causes 'pathological' heart rate turbulence. Am J Physiol Regul Integr Comp Physiol 2000; 279: R1171-R1175.

5. Schmidt G, Malik M, Barthel P, Schneider R, Ulm K, Rolnitzky L, et al. Heart-rate turbulence after ventricular premature beats as a predictor of mortality after acute myocardial infarction. Lancet 1999 ; 353: $1390-1396$

6. Ghuran A, Reid F, La Rovere MT, Schmidt G, Bigger JT Jr, Camm AJ, et al. The ATRAMI Investigators: Heart rate turbulence-based predictors of fatal and nonfatal cardiac arrest (The Autonomic Tone and Reflexes After Myocardial Infarction substudy). Am J Cardiol 2002; 89: $184-190$
7. Koyama J, Watanabe J, Yamada A, Koseki Y, Konno Y, Toda S, et al. Evaluation of heart-rate turbulence as a new prognostic marker in patients with chronic heart failure. Circ J 2002; 66: 902-907.

8. Maron BJ, Olivotto I, Bellone P, Conte MR, Cecchi F, Flygenring $\mathrm{BP}$, et al. Clinical profile of stroke in 900 patients with hypertrophic cardiomyopathy. J Am Coll Cardiol 2002; 39: 301-307.

9. Klues HG, Schiffers A, Maron BJ. Phenotypic spectrum and patterns of left ventricular hypertrophy in hypertrophic cardiomyopathy: Morphologic observations and significance as assessed by twodimensional echocardiography in 600 patients. $J$ Am Coll Cardiol 1995; 26: $1699-1708$.

10. Davies LC, Francis DP, Ponikowski P, Piepoli MF, Coats AJ. Relation of heart rate and blood pressure turbulence following premature ventricular complexes to baroreflex sensitivity in chronic congestive heart failure. Am J Cardiol 2001; 87: 737-742.

11. Lin LY, Lai LP, Lin JL, Du CC, Shau WY, Chan HL, et al. Tight mechanism correlation between heart rate turbulence and baroreflex sensitivity: Sequential autonomic blockade analysis. J Cardiovasc Electrophysiol 2002; 13: 427-431.

12. Thomson HL, Morris-Thurgood J, Atherton J, Frenneaux M. Reduced cardiopulmonary baroreflex sensitivity in patients with hypertrophic cardiomyopathy. J Am Coll Cardiol 1998; 31: 1377-1382.

13. Gilligan DM, Nihoyannopoulos P, Chan WL, Oakley CM. Investigation of a hemodynamic basis for syncope in hypertrophic cardiomyopathy. Circulation 1992; 85: 2140-2148. 